\title{
CONTAMOS TRABAJADORES O CONTAMOS CON LOS TRABAJADORES. TRABAJO Y EMPRESA EN LA ESPAÑA CONTEMPORÁNEA
}

\author{
LINA GÁLVEZ MUÑOZ * \\ Universidad de Reading
}

\section{RESUMEN}

En este artículo se reflexiona sobre la necesidad de crear un marco analítico específico que comprenda el trabajo como un factor esencial dentro del conjunto de las relaciones internas y externas que definen la competitividad de las empresas y las estrategias que éstas desarrollaron para adaptarse al medio. Se centra en el caso español, atendiendo a las diversidades regionales y sectoriales. Frente al concepto de trabajo como fuerza comprada y aplicada, y al estudio del conflicto entre trabajadores y empresarios, en este artículo se analiza la evolución de las formas de organización del trabajo y se considera al trabajo como un elemento que puede incentivar, impedir, facilitar, retrasar y, sobre todo, adaptar a las distintas condiciones de partida el cambio tecnológico. Frente al énfasis en el capital físico a la hora de explicar las fuentes de competitividad de las empresas, se incide en la importancia del capital humano, sobre todo de las cualificaciones adquiridas en los talleres y de las cualificaciones organizativas y de investigación. Especial atención se presta al contexto institucional y a la interacción de las empresas con el Estado en materia laboral en su triple papel de empleador, regulador e intermediario en la transmisión de cualificaciones y conocimientos.

\section{ABSTRACT}

This article tries to provide a specific analytical framework which includes labour as an essential factor within the internal and external set of relations defining the competitiveness of firms and their strategies to adapt to the envi-

* Quisiera agradecer los comentarios de dos evaluadores anónimos y de los comen. taristas en el Seminario de Cuenca - Albert Carreras y Carles Sudrià- a la primera versión de este artículo que triplicaba en espacio y referencias al presente trabajo, ya que se trataba de un trabajo historiográfico. Quisiera también expresar mi agradecimiento a Joaquim Cuevas, Santiago López, José Ignacio Martínez, Teresa Muñoz y Javier Vidal, y dejar constancia de que ninguno de ellos es responsable de los posibles errores de este artículo. 
ronment in which they operate. Special attention is given to the Spanish case, taking into consideration its regional and sectorial diversity. Instead of studying labour as an input to be bought and applied, or the history of the conflict between employers and employees, this article analyses the evolution of how different forms labour have been organised within firms. Labour is considered as an element with the potential to provide an incentive, to impede, facilitate, delay, and mainly adapt technological change to the different factor endowments. The emphasis is put on human capital -mainly shopfloor skills, organisation skills and research skills - instead of physical capital to explain the competitiveness of firms. Special attention is reserved to the institutional context and the interaction between business and governments on labour issues, analysing the state as an employer, regulator and intermediary in the transmission of skills and knowledge.

\section{INTRODUCCIÓN}

Dentro de una empresa, el proceso de creación de valor comprende una división especializada del trabajo que es tanto jerárquica como técnica y que se basa en las capacidades cognitivas y físicas de los trabajadores, conocidas como cualificaciones. La transformación de esas cualificaciones en productos requiere que los trabajadores aporten esfuerzo al proceso productivo, y que los gestores organicen la combinación de inputs de producción de la forma más eficiente posible en relación con los mercados a los que quieren abastecer, el marco institucional en el que operan y la tecnología de la que disponen dentro de un sistema tecno-laboral que es idiosincrásico a cada empresa ${ }^{1}$. Por tanto, sin analizar cómo las empresas organizan $y$, sobre todo, han organizado a sus trabajadores para transformar los recursos invertidos en bienes vendibles, es imposible comprender la naturaleza de la competitividad de las mismas, los determinantes e impacto social del cambio tecnológico $\mathrm{y}$, consecuentemente, no puede tampoco entenderse la dinámica del desarrollo capitalista, ni las distintas formas y ritmos en que éste ha evolucionado en cada sector, región y país. Desde que la tecnología comenzó a considerarse como un sistema, y los problemas tecnológicos como problemas tecno-económicos, el factor trabajo se ha situado en el centro del cambio tecnológico, pero no exclusivamente en

1 Para Lipartito (1994), la tecnología no es un bien público ni un artefacto, es un bien específico que tiene que ser aplicado en el lugar de trabajo. Un sistema técnico socialmente construido incluye una mano de obra socialmente construida, por lo que es preferible hablar de sistemas tecno-laborales que de sistemas tecnológicos. Sobre las ventajas comparativas relacionadas con la distinta organización de los talleres, véase Lazonick (1990). 
relación con el control que los empresarios buscan de este factor de producción, sino como un elemento complejo que puede incentivar, impedir, facilitar, retrasar y, sobre todo, adaptar a las distintas condiciones locales de partida el cambio tecnológico, a través de lo que Mokyr (1990) ha llamado micro-invenciones, es decir, las adaptaciones basadas en el método de prueba y error. Por tanto, para explicar las diferencias en la productividad se pone el énfasis en el capital humano -principalmente en las cualificaciones adquiridas en los talleres, las cualificaciones organizativas y las de investigación frente a los índices de alfabetización- y en las formas de organización de la producción por encima del capital físico.

El énfasis del lado de la oferta - aunque nunca se puede disociar de los factores de demanda-, coincide con el protagonismo que a la empresa, en cuanto centro del cambio tecnológico, le confieren marcos teóricos como la economía evolutiva y la de recursos y capacidades. De esta forma, se puede profundizar en el análisis de la competitividad de las empresas y las industrias, así como en el análisis de los niveles de productividad a escala sectorial, regional, nacional o internacional. No es, pues, de extrañar que los estudios que tratan de analizar la competitividad internacional de las industrias de determinados países o regiones a través de la medición de los distintos niveles de productividad comiencen a hacer hincapié en la necesidad de medir la productividad a través de una ecuación de productividad total de los factores, ya que, puesto que la productividad del trabajo será mayor en aquellos países o regiones intensivos en capital, aquélla no es una medida adecuada de eficiencia. Estos enfoques llevan implícita, aunque a veces se hace explícita, la consideración de la organización del trabajo como una variable explicativa ${ }^{2}$. Esto se encuentra en consonancia con el análisis histórico sobre la organización del trabajo, que muestra que no siempre la producción en masa con mayores índices de productividad laboral fue más eficiente que la producción flexible, ya que la especialización flexible, principalmente organizada en pequeñas y medianas empresas normalmente agrupadas en distritos industriales, tuvo en muchos sectores mayor importancia que la producción en masa, por su contribución tanto al empleo como al valor añadido industrial ${ }^{3}$. Sin embargo, todavía es difícil cuantificar esta variable. Primero, porque para sumar hay que encontrar un común denominador y la evidencia que hasta ahora se tiene tiende a señalar la diversidad como norma, por lo que la variable

${ }^{2}$ Sobre España, véanse las aportaciones en este número de Joan Ramón Rosés, y de Mar Cebrián.

${ }^{3}$ Scranton (1991) y Becatini (1987). 
tendría que ser calculada independientemente y esto es algo que aún no se ha llevado a cabo; segundo, porque aunque la abundancia relativa de los factores de producción determina el tipo de tecnología empleado, los factores institucionales y sociales deben de tenerse en cuenta, y, finalmente, porque aún faltan estudios a nivel micro que aporten información sobre la interacción entre la productividad de las empresas y la organización del trabajo en el seno de éstas. Es en este tercer punto en el que se concentra este artículo para llamar la atención sobre la necesidad de «contar con los trabajadores» además de contarlos, en tener un marco analítico específico que comprenda el trabajo como un factor esencial dentro del conjunto de relaciones internas y externas de la empresa que definan la competitividad de las empresas distinguiendo las condiciones particulares de cada caso o región y siempre dentro de su marco institucional. Especialmente interesante resulta la interacción entre empresa y Estado y la evolución del reparto de competencias entre ambos en materia laboral. Si se parte de la idea de que el trabajo es la transformación de cualificaciones en productos, deja de ser el interés principal de este artículo el concepto de trabajo como fuerza comprada y aplicada empleado por los historiadores económicos, así como también la historia sindical y la del movimiento obrero según son tratadas por los historiadores sociales, ya que desde esta perspectiva se desemboca casi automáticamente en la historia del conflicto. Lo que interesa es el modelo de organización que es diseñado por el empresario, el ejecutivo u otros asalariados para hacer que las cualificaciones se transformen en productos. Son relevantes, por tanto, los momentos en que cambian los diseños y los procesos para producir, en particular cuando los asalariados participan, o quieren participar, del poder de decisión de la empresa sobre las formas de organización empresarial a las que dan lugar con su actuación, de manera formal o informal, a través de la vía sindical o no.

Estos aspectos han sido tratados por la sociología del trabajo y los estudios de organización empresarial. Y aunque la perspectiva histórica suele respetarse y valorarse, estos estudios se concentran, en su mayoría, en el análisis de los paradigmas organizativos a nivel general y a partir de una base documental principalmente bibliográfica y oral, frente al uso más intensivo de los archivos que hacen los historiadores y que les permite comprobar la aplicación efectiva de esos paradigmas de organización. No obstante, el tratamiento de la organización del trabajo dentro de las empresas aún no ha sido integrado de forma satisfactoria en los estudios de 
historia empresarial ${ }^{4}$. La necesidad de integrar los aspectos laborales en el centro de los estudios de historia empresarial se torna más urgente, sobre todo teniendo en cuenta el protagonismo que marcos teóricos como la economía evolutiva y la de recursos y capacidades conceden a la dinámica de las organizaciones empresariales y, por tanto, a la historia económica de la empresa. Esta urgencia se incrementa en historiografías en las que la historia empresarial se ha desarrollado tardíamente y puede contar con las ventajas relacionadas con los late-comers, como es el caso de la española ${ }^{5}$. Sin embargo, son pocos los estudios dentro de la historiografía empresarial española que «cuentan con los trabajadores». El escaso tratamiento del factor trabajo dentro de la empresa parece especialmente grave en el caso español, si se acepta el argumento de Guillén de que uno de los mayores problemas con los que contó la industria española, y que resulta fundamental a la hora de explicar su retraso, fue la falta de implantación de métodos de gestión modernos, dada la preocupación de los empresarios por mantener la paz social aun por encima de la eficiencia en la producción ${ }^{6}$. En este sentido, habría que prestar igualmente atención al peso que ha tenido la empresa pública en la historia de España en comparación con otros países, sobre todo durante la etapa del franquismo, cuando lo que Carreras y Tafunell llaman empresas nacionales -empresas públicas con pocos beneficios que no seguían criterios de eficiencia- abundaban y estaban fuertemente relacionadas con la necesidad de pleno empleo y, por ende, de paz social ${ }^{7}$. Por todo ello, este artículo se divide en dos partes fundamentales. En la primera se desarrolla una evolución a grandes rasgos de la organización del trabajo dentro de las empresas, con el fin de apuntar las ventajas que la integración del factor trabajo dentro de la historia empresarial reportaría al conocimiento del pasado económico. En la segunda se llama la atención sobre el escaso tratamiento que este enfoque ha tenido en la historiografía española y se intentan ofrecer posibles vías de integración, principalmente, el papel desempeñado por el Estado en la organización de la producción de las empresas, en su triple papel de empleador, regulador e intermediario, no tanto en materia de negociaciones colectivas como en calidad de impulsor y difusor de la formación y el conocimiento.

4 Scranton y Horowitz (1997) y Fitzgerald y Rowley (1997).

'Esta posible ventaja comparativa ya ha sido comentada por Valdaliso (1993), Segreto (1994) y Martínez Ruiz (1998), en el caso de este último en relación con la integración del factor trabajo.

"Guillén (1994).

${ }^{7}$ Carreras y Tafunell (1997). 


\section{LA EVOLUCIÓN DE LA ORGANIZACIÓN DEL TRABAJO DENTRO DE LAS EMPRESAS}

Los cambios en la organización del trabajo dentro de las empresas han estado básicamente encaminados a conseguir la mayor eficiencia posible mediante el incremento de la productividad, ya fuera a través de la reducción de insumos o de la creación de mayor valor añadido por unidad de producto. $Y$ han estado principalmente ligados con otros dos aspectos: la modificación del marco tecnológico y la evolución del marco institucional de las relaciones laborales y la intervención estatal. Esto está relacionado con las diferencias que se establecen en los procesos de difusión tecnológica, donde los gestores y los ingenieros no tienen normalmente un control absoluto sobre las innovaciones, sino que actúan con conocimiento imperfecto y tienden a concentrar sus esfuerzos en incrementar los resultados de la tecnología existente, evitando un cambio brusco, siempre difícil de imponer, y construyendo así sistemas específicos que siguen fuertes tendencias históricas, derivadas de eventos o elecciones previas, y que reflejan el equilibrio entre los intereses de los empresarios, los trabajadores, los consumidores y los políticos. Esto, además, explicaría la convivencia de distintas etapas de cambio tecnológico incluso dentro de una misma empresa. Los gestores de las empresas buscan relaciones estables entre la tecnología de la producción, la organización del trabajo y la cultura de la mano de obra. Distintas combinaciones de estos tres factores pueden considerarse productivas para una determinada industria, pero las elegidas en un momento dado tienden a mantenerse. Los gobiernos también han tenido un papel esencial en la búsqueda de ese equilibrio ${ }^{8}$. Siguiendo el argumento de Guillén de que en España, los empresarios, temerosos del enfrentamiento con los obreros, adaptaron tardiamente técnicas modernas de organización de la producción, lo que incidió en agravar la falta de competitividad de la empresa española, puede decirse, que la búsqueda de ese equilibrio pudo ser más acusada que en otros países ${ }^{9}$. Además, Riera insiste en que los inge-

${ }^{*}$ Esto queda de manifiesto en los manuales usados por la Comisión Nacional de Productividad Industrial (CNPI) creada conforme al Decreto del 1 de mayo de 1952 por el gobierno de Franco, para solucionar el problema de productividad, como queda recogido a continuación: «Cualquier programa que se emprenda para mejorar el rendimiento de una industria precisa un estudio racional de los métodos de trabajo en ella empleados, con el fin de conseguir el máximo aprovechamiento tanto del hombre como de los medios de que la empresa dispone para la producción, así como una disminución de sus costes y una mejora en la calidad, todo ello sin la necesidad de nuevas inversiones, salvo en aquello que sea verdaderamente necesario.» Citado en Buesa y Molero (1982), p. 258.

9 Guillén (1994). 
nieros españoles desempeñaron el papel que les otorgó la burguesía como intermediarios entre ésta y el proletariado, por lo que, a lo largo del siglo xx, estuvieron más preocupados por las cuestiones sociales que por las técnicas, adoptando las más de las veces posturas paternalistas frente a las actitudes más «científicas» que se podían esperar, dada su formación ${ }^{10}$. Pero resulta difícil definir, sobre todo a la hora de compararlo con otros países de su entorno, lo que ocurrió en las industrias españolas; saber hasta qué punto esa tendencia a la continuidad que se apunta en la bibliografía fue fruto del miedo al obrero, de la intervención estatal o de una utilización de los recursos conforme con las condiciones de partida del país, donde el factor trabajo era abundante pero estaba caracterizado por su bajo capital humano en muchos sectores y regiones ${ }^{11}$.

El triunfo de la fábrica trajo la imposición de la disciplina fabril, ya fuera por cuestiones de eficiencia relacionadas con las nuevas tecnologías, con el ahorro en costes de transacción, o con el control sobre la mano de obra. Pero la difusión del sistema fabril fue lenta y desigual, y, de hecho, la revolución industrial no sólo aumentó las posibilidades laborales en las fábricas, sino que, incluso en Inglaterra, multiplicó las oportunidades dentro de la industria doméstica y los pequeños talleres, donde los trabajadores escaparon a la disciplina fabril. Además, la diversidad de modelos organizativos y de tecnologías empleadas fue mucho más acentuada en los países seguidores, y no sólo dependía de la industria, sino que, a veces, existía dentro de un mismo sector industrial. Esta diversidad de tecnologías y sistemas productivos está íntimamente relacionada con el tipo de producto y el tamaño del mercado al que va dirigido (factores que condicionan la escala, la tecnología empleada y el coste medio de producción) y con las estructuras sociales e institucionales predominantes. De hecho, la pluralidad de estructuras industriales debe ser explicada no sólo en términos de fuerzas de mercado, sino también, y sobre todo, en función de las instituciones y las estructuras sociales predominantes dentro de industrias y regiones concretas ${ }^{12}$. En España, por ejemplo, las mayores concentraciones fabriles se dieron, desde principios del siglo XIX, en la industria del tabaco, en la cual las trabajadoras escaparon de la organización de la producción impuesta a través de la disciplina fabril, a pesar de que algunas fábricas, como la de Sevilla, superaron las 7.000 trabajadoras durante ese período.

\footnotetext{
10 Riera (1993).

"Veáse la aportación de Rosés en este volumen; sobre el capital humano en general en España, Núñez (1992).

12 Valdaliso y López (2000), pp. 183-184.
} 
La producción del tabaco en polvo, dominante durante el siglo xv山 y que era intensiva en capital, fue sustituida en el siglo xxx por la elaboración de tabaco de humo, que era intensiva en trabajo y que requería la utilización de una mano de obra cualificada que bien podía haberse organizado en pequeños talleres ${ }^{13}$. La concentración de la producción no estaba relacionada con necesidades de la producción, ya que las economías de escala fueron pocas durante el siglo XIX y estaban concentradas en las primeras fases del proceso de producción, que comenzaron a mecanizarse a mediados de siglo, sino con necesidades fiscales derivadas del carácter de monopolio fiscal de la industria del tabaco española. Esto explica que se mantuviese una producción flexible con una mano de obra no sujeta a la disciplina fabril pero concentrada en grandes fábricas.

Los cambios tecnológicos y en la organización de la producción fueron unidos a importantes cambios institucionales, tales como la libertad para factores de producción, entre ellos el trabajo, a través de la abolición de los gremios, aunque fue posteriormente, a raíz de la segunda revolución industrial, cuando comenzó a desarrollarse una legislación social en paralelo al desarrollo de los sindicatos ${ }^{14}$. Esta legislación estaba encaminada, en un principio, a proteger al obrero, individualmente, y a la sociedad, colectivamente, de la presión obrera, y tuvo más un carácter normativo que práctico. Para Rivera supuso, además, el paso del abstencionismo normativo por parte del Estado a su decidida intervención en materia social ${ }^{15}$. Durante esta «primera ruptura industrial», la difusión de nuevas tecnologías trajo de nuevo aparejados cambios en la organización de la producción que hasta muy recientemente se habían identificado con la aparición de la gran empresa y la producción en masa. Aunque el carácter de capital intensivo de muchos sectores aumentó las barreras de entrada y acabó consolidando una estructura monopolística u oligopolística, la especialización flexible siguió siendo tanto o más importante que la producción en masa. No obstante, comenzó a extenderse el concepto de racionalización y de superioridad y mayor eficiencia de la producción en masa. De hecho, la utilización del sistema de producción en masa en los Estados Unidos, como el taylorismo y el fordismo, donde el concepto de racionalización era esencial, se ha considerado como una variable de alto valor explicativo a la

${ }^{13}$ Para Gálvez (2000b) la razón es de tipo institucional.

14 En España los gremios quedaron suprimidos por las Cortes de Cádiz en 1813, pero esto no se hizo efectivo hasta la vuelta definitiva de los liberales al poder en 1835 .

${ }^{15}$ Rivera (1998). 
hora de entender por qué la productividad americana duplicó el nivel de la inglesa entre 1870 y $1940^{16}$. Sin embargo, de nuevo es necesario llamar la atención sobre las diferencias que marca, en los cálculos de productividad del trabajo, la consideración de la organización del trabajo en relación con los recursos de que se dispone y el marco institucional en el que se opera.

En España, la producción en masa y los dos sistemas de organización de la producción aparejados a ella, el taylorismo u organización cientifica del trabajo y el fordismo, no tuvieron una implantación efectiva hasta la posguerra, y aun entonces, y a pesar del fuerte apoyo institucional recibido con la creación en 1946 del Instituto de Racionalización del Trabajo por el régimen de Franco, su aplicación fue limitada ${ }^{17}$. De hecho, una de las principales causas que apuntan para explicar la falta de implantación de la organización científica del trabajo con anterioridad a los años cuarenta y cincuenta es la falta de apoyo institucional ${ }^{18}$. Otras causas señaladas son un cierto aislacionismo internacional y una marcada mentalidad antimodernista muy relacionada con el pensamiento católico, que veía en la equiparación del obrero a la máquina implícita en el taylorismo una degradación moral de la persona. Se insiste en identificar como elemento clave la búsqueda de la paz social, que llevó a empresarios y gerentes a adoptar cualquier método que la garantizase aun a costa de una mayor eficiencia. Igualmente, son destacables la escasez de ingenieros, auténticos promotores y difusores del taylorimo junto con las consultorías especializadas que también comienzan a aparecer en esos años, y el poco desarrollo tecnológico de las empresas en España, que hacía difícil introducir métodos de producción en masa y prescindir de los trabajadores cualificados sustituyéndolos por otros no cualificados. $Y$ en consonancia con todo lo expuesto, no hay que olvidar la falta de burocratización de las empresas españolas, en parte atribuible a su menor tamaño. Aunque en España existía la gran empresa, y ésta normalmente dominaba los mercados de los sectores en los que operaba, y contaba, como en el caso del tabaco o de los ferrocarriles, con un importante cuerpo de ingenieros, la organización científica del trabajo no logró introducirse de forma importante hasta el régimen franquista ${ }^{19}$. En el caso de la Compañía Arrendataria de Tabacos, que gestionó el monopolio público de tabacos entre 1887 y 1945, el monopolio y el

16 Tolliday y Zeitlin (1991) y Broadberry (1997).

17 Guillén (1994) y Buesa y Molero (1982).

${ }^{1 *}$ Guillén (1994).

14 Carreras y Tafunell (1997). 
mantenimiento de una mano de obra femenina que aceptaba la flexibilidad en mayor medida que la masculina, y a la que incentivaban a la asistencia o no dependiendo de las necesidades de la producción, permitieron a la compañía mecanizar la producción sin introducir métodos tayloristas que hubieran generado expulsiones masivas de personal, algo que hubiera sido ineficiente para una compañía gestora de un monopolio fiscal por el coste político que ello habría supuesto ${ }^{20}$.

Un sistema de gestión es, por un lado, una tarea técnica de organización y, por el otro, un sistema de autoridad jerárquica. Los gestores imponen nuevos métodos de organización para afrontar los problemas ideológicos y técnicos que aparecen. Cuando se producen cambios en el tamaño y la complejidad de las empresas, aumenta la competencia internacional o se está bajo los efectos de las reivindicaciones obreras, los teóricos de la organización industrial y los practicantes tratan de imponer nuevos sistemas, aunque también puede ocurrir que sólo los modifiquen. El eclecticismo y el paternalismo parecen haber sido las respuestas de los empresarios españoles a la adaptación de las prácticas de gestión con anterioridad al régimen franquista, pero faltan observaciones que ayuden a conocer cómo se difundieron los paradigmas de organización en las distintas regiones y sectores económicos, y cómo esto interactuó con el tejido industrial y los condicionamientos institucionales y culturales ${ }^{21}$. Y sin una comprensión previa de cómo se difundieron los paradigmas de organización de la producción y del trabajo es difícil entender plenamente cómo se llevó a cabo la difusión tecnológica, ya que ésta cuenta con una parte informativa, la más pública, y otra más específica e indicativa de cómo deben de hacerse las cosas. Estudios que insistan en el origen económico del paternalismo, en que la filantropía marchaba de acuerdo con el interés industrial, son esenciales para entender su dimensión como sistema de gestión empre-

${ }^{20}$ Para la Compañía Arrendataria de Tabacos, Gálvez (2000a); Bowden y Higgins (1999), tratan de unir el debate sobre las empresas algodoneras desarrollado dentro de la historia empresarial con el debate sobre los niveles de productividad nacional desarrollado dentro de la historia económica, llegando a la conclusión de que los limitados cambios que se produjeron eran un reflejo de la comprensible, aunque costosa, predisposición a sacrificar los esquemas de mejoras en la productividad con potencial a largo plazo ante los más modestos esquemas de conservar el consenso y la solidaridad a corto plazo.

${ }^{21}$ De hecho, una de las mayores críticas que se le han hecho a Guillén (1994) es la falta de trabajo de archivo para documentar bien su hipótesis y estudiar la repercusión de los distintos modelos de gestión en las empresas. Valdaliso (1999), p. 14. 
sarial ${ }^{22}$. Aproximarse a los orígenes económicos del paternalismo y, por tanto, considerarlo como sistema de gestión empresarial permite retomar la idea de que la eficiencia de los sistemas de organización debe ser analizada en relación con las diferentes condiciones de partida, tanto económicas como institucionales y culturales. En este sentido, se explica por qué se considera como una estrategia eficiente la especialización flexible que siguieron países como Alemania tras la Segunda Guerra Mundial, mientras que la adopción de métodos de producción en masa en la industria británica a través de la americanización de su tejido industrial se encuentra, según Jones, en la raíz de la pérdida de competitividad de esta industria ${ }^{23}$. En España, la introducción de los métodos de producción en masa y la organización científica del trabajo se concentran en el primer franquismo, y se insertan, como en el resto de Europa, en el marco de una insoslayable preocupación por la productividad y una mayor intervención del Estado en la economía tras la Segunda Guerra Mundial. Así como dentro de los efectos de la ayuda americana, la cual no sólo se concentró en la trans- ferencia de bienes físicos, sino también en la difusión de las prácticas de organización americanas a través del fomento de instituciones especializadas, como la ya mencionada CNPI o la Asociación para el Progreso de la Dirección (APD), creada en 1956, y de labores de consultoría e intercambios y visitas a Estados Unidos ${ }^{24}$. La racionalización encontró un caldo de cultivo especialmente bueno, algo que no había ocurrido con anterioridad, en el nuevo Estado franquista, tanto por el papel que comenzaron a representar los ingenieros en las empresas y la política económica como por la instauración de un sistema de relaciones laborales basado en el sindicato vertical, al que todos los obreros y empresarios de un sector debían pertenecer, dentro de un marco institucional represivo para las rela-

${ }^{22}$ Aunque los estudios sobre el paternalismo abundan en la historiografía española, éste se ha estudiado como una respuesta a la reforma social, más que como un sistema de organización de la producción. Estudios como el de Aubanell (1998) sobre las prácticas paternalistas y la implantación del sistema de bienestar industrial en las eléctricas madrileñas, no son frecuentes. Este sistema queda definido como la provisión sistemática - frente a la personal que representa el paternalismo- de un servicio independientemente del salario por parte de la empresa al personal de ésta. Véase también Babiano (1998).

${ }^{23}$ Jones (1997).

24 Sobre el papel de los Estados Unidos en el movimiento de la productividad en España véase Gil (1967). Los esfuerzos por incrementar la productividad en la posguerra también se vivieron en otros países. Especialmente interesante resulta el caso británico estudiado por Tiratsoo y Tomlinson (1993), donde los esfuerzos del gobierno para diseminar el conocimiento encaminado a la mejora de la productividad se vieron truncados por la desconfianza de las empresas hacia la intervención estatal. 
ciones laborales. Además, hay que tener en cuenta que los cambios se introducen más rápidamente en estructuras jerárquicas donde la toma de decisiones va de arriba a abajo. En este orden de cosas, no es de extrañar que fueran principalmente las empresas del INI las que introdujesen las técnicas tayloristas. Babiano ha encontrado en la militarización y el paternalismo dos elementos claves a la hora de explicar el «fordismo a la española» ${ }^{25}$.

La legitimidad económica que concedió el apoyo del régimen franquista a la imposición de este sistema se basó en tres pilares fundamentales: que la cuestión de la productividad respondía tanto a los intereses empresariales individuales como a los generales del pais; que la mejora de la productividad tenía un carácter técnico-económico en el que se combinaban múltiples variables, entre ellas el aprovechamiento del capital físico y humano existente, $y$, finalmente, que el objetivo último de la aplicación de la organización científica del trabajo - y posteriormente también de los métodos de la escuela de relaciones humanas - era la consecución del bienestar colectivo de empresarios y trabajadores, derivado de los beneficios que el incremento de la productividad comportaba ${ }^{26}$. No obstante, no hay que olvidar que las relaciones laborales durante el franquismo se caracterizaron por la falta de libertad y de flexibilidad, y por el control por parte de los empresarios, aunque con una fuerte intromisión estatal. El fuero del trabajo de 1938 permitió controles administrativos en los salarios y en las condiciones de trabajo, afianzando el paternalismo estatal y, por tanto, la intromisión del Estado en la organización del trabajo dentro de las empresas, aunque también reconocía el derecho de los empresarios a controlar la empresa, claro que frente a los trabajadores. En este sentido, no es de extrañar, como constatan De Miguel y Linz, que en los años sesenta los empresarios españoles reclamaran un menor control estatal y una mayor flexibilidad en el despido, aunque para Babiano, la rigidez de las relaciones laborales no es una peculiaridad de las instituciones y leyes franquistas, sino una de las piezas del puzzle fordiano, en general ${ }^{27}$. No obstante,

${ }^{25}$ Babiano (1998). Guillén (1994), p. 177. Castillo (1996), p. 240, denomina la Dictadura como «ambiente para los organizadores», aunque en este caso, se refiere a la extensión de las ideas tayloristas durante la dictadura de Primo de Rivera. Lozano (1996), en su investigación sobre los arsenales españoles, apunta cómo en los establecimientos militares los cambios se imponían con mucha más velocidad que en los civiles lo que seguramente haya que relacionarlo con el principio de jerarquía que es inherente a la institución castrense. Esto es consecuente con la importancia que Babiano concede a la militarización de las relaciones laborales en España durante el franquismo.

${ }^{26}$ Buesa y Molero (1982), p. 258.

${ }^{27}$ De Miguel y Linz (1963), Babiano (1998). 
las relaciones laborales siguieron siendo un problema básico para las empresas españolas a la hora de implantar métodos de gestión modernos. El crecimiento económico experimentado en los años sesenta aumentó la complejidad en las empresas españolas, que por fin alcanzaron los niveles de burocratización que tenían las alemanas y americanas en los años veinte, período en el que la organización científica del trabajo se impuso en esos países. Puesto que para Guillén, la escasa burocratización se ha considerado una pieza clave a la hora de explicar la falta de implantación de paradigmas de organización en estado puro, como el taylorismo, frente a la postura ecléctica tomada por los empresarios españoles, cabría esperar que éstos comenzaran a implantarse con más éxito durante los años sesenta, aunque siempre teniendo en cuenta que el análisis histórico tiende a demostrar que los paradigmas se adaptan más que implantarse en estado puro ${ }^{28}$. En estos años fue el modelo de relaciones humanas el que comenzó a introducirse, de nuevo con un claro apoyo estatal unido con el fomento de la ayuda americana, como es el caso también de la organización científica del trabajo, pero gozando el primero de una mayor aceptación social debido a su mayor concordancia con la mentalidad tradicional, católica y humanista predominante en España, aunque la implantación práctica de este método se tradujo más en la adopción de un lenguaje que en una realidad. De nuevo, hay que llamar la atención sobre la falta en la historiografía económica de la empresa de estudios de caso que lo constaten. La creciente conflictividad social y el riesgo político pueden explicar en parte por qué los empresarios españoles siguieron adoptando prácticas autoritarias y paternalistas, que llevan a identificar aún hoy en día, en estudios sobre los métodos de gestión comparados a nivel internacional, el Spanish management - si es que tal generalización puede hacerse- con el autoritarismo y el paternalismo ${ }^{29}$. Los dos tipos de empresas predominantes en España desde los años del franquismo hasta mediados de los años ochenta, cuando comienzan la venta de empresas del INI y los procesos de privatización, son la empresa familiar y la empresa pública. La falta de personal especializado en la gestión de empresas puede explicar esta falta de profesionalidad y de implantación de métodos de gestión estandarizados frente a la generalización de las prácticas paternalistas. La proliferación en España de lo que se conoce como canales de difusión del conocimiento de organización de empresas (las escuelas de empresa, las publicaciones, las con-

\footnotetext{
${ }^{28}$ Guillén (1994).

${ }^{29}$ Por ejemplo, en Bruton (1994).
} 
sultorías y las multinacionales), así como la apertura a la competencia exterior, y la mayor formación de la población son fundamentales para explicar el proceso de profesionalización de los métodos de gestión al que se asiste hoy en día. Aun así, durante la democracia las empresas españolas han seguido sufriendo las consecuencias de un entorno institucional fuertemente burocratizado y lento que también afecta a las relaciones laborales, las cuales continúan distinguiéndose por las prácticas de gestión autoritaria frente a la mayor incorporación de los trabajadores a la toma de decisiones empresariales observable en otros paises, donde, además, la formación de los trabajadores dentro de las empresas ha pasado a tener una mayor importancia. Esto hay que relacionarlo con los nuevos avances tecnológicos, la «segunda ruptura industrial» y la fuerza recobrada de los métodos de producción flexibles como resultado de la revolución de la información ${ }^{30}$. Todavía están por ver las consecuencias de las privatizaciones en la introducción de prácticas de gestión menos paternalistas. No obstante, no debe olvidarse que aún se mantiene una importante economía sumergida y que el modo de producción doméstico sigue siendo importante en muchos sectores como el textil o el del calzado, donde el trabajo sigue organizándose como el sweating system, ya que España continúa teniendo abundancia de mano de obra sin cualificar.

\section{LA FALTA DE INTEGRACIÓN DEL FACTOR TRABAJO EN LA HISTORIA EMPRESARIAL ESPAÑOLA}

Dos conjuntos de razones explican la falta de estudios sobre la organización del trabajo dentro de la historia empresarial española. Por una parte, se encuentra el desarrollo tardío de esta disciplina, y, por otra, la evolución del reparto de competencias entre el Estado y las empresas en materia laboral.

Con respecto a la primera razón, la historia empresarial comenzó a consolidarse como una disciplina autónoma en parte gracias a la obra de Alfred D. Chandler Jr., que, además, la llevó más allá de sus propias fron-

${ }^{30}$ Véase Tolliday y Zeitlin (1991). Sobre la falta de flexibilidad que sufren las empresas españolas, véase el informe sobre España de la OCDE de 1998. Malo Ocaña (1999) ha estimado las diferencias del coste del despido individual en la dictadura y en la democracia en España, llegando a la conclusión de que los costes del despido no disminuyeron con el cambio de sistema en las relaciones labores propiciado por la democracia, ya que las indemnizaciones medias fueron más elevadas en este último, ya incluso antes de la generalización de los contratos temporales a partir de 1984. 
teras. Su principal aportación no sólo ha sido la de proporcionar una constatación del crecimiento y desarrollo de la gran empresa americana y, posteriormente, británica y alemana, sino también la de desarrollar un paradigma, un conjunto de proposiciones acerca del desarrollo de la empresa y de las fuentes de sus ventajas comparativas. Aunque Chandler revolucionó el estudio de la historia empresarial, deshumanizó la empresa. Ni los empresarios ni los trabajadores aparecen en el análisis de Chandler, y los gestores, la mano visible del cambio, que sí lo hacen, son meros asalariados de los que no interesa ni su origen ni su evolución, sino qué clase de decisiones tomaban y por qué. Éste ha sido el paradigma principal de análisis sobre el que se ha desarrollado la historia empresarial en España, aunque se puede decir que de forma muy tardía, y que han sido principalmente los estudios realizados dentro de la historia económica con perspectivas sectoriales o regionales - una de las líneas de investigación más fructíferas de esta disciplina- los que han permitido realmente incrementar los conocimientos que se tienen sobre las empresas, los empresarios y la estructura y evolución de los diferentes sectores económicos ${ }^{31}$. Quizás hayan sido los modernistas, como Torras, los que mejor han sabido situar el factor trabajo en el corazón del cambio industrial y empresarial ${ }^{32}$. Sin embargo, una vez que el trabajo puede ser contratado libremente en el mercado y aparecen los sindicatos, las cuestiones laborales desaparecen del núcleo del análisis empresarial. Sólo algunas historias de empresa incluyen a los trabajadores, aunque normalmente lo hacen en capítulos aparte, como si el trabajo y las relaciones laborales fuesen algo interno de la empresa pero al mismo tiempo externo, separado. Aun así, proporcionan datos básicos sobre los niveles de vida, la movilidad laboral y las prácticas de reclutamiento de la mano de obra ${ }^{33}$. Las aportaciones más importantes de la historia empresarial en España se han reunido principalmente en los documentos de trabajo de la Fundación Empresa Pública y en varias obras colectivas, donde pocos autores tratan los aspectos laborales de las empresas

11 Valdaliso (1999), p. 8. Especialmente interesantes para conocer la relación entre el cambio tecnológico y las características de la mano de obra y la innovación organizativa en distintos sectores industriales son los trabajos de Lozano (1997), Rosés (1997), Muñoz (1999) y Gálvez (2000a).

32 Torras (1993).

33 También existen historias de empresa en las que el factor trabajo está integrado en el discurso general de la misma, como Comin y Martín Aceña (1999) o Houpt y Ortiz Villajos (1998). En otros, la historia de la empresa se ha hecho desde la perspectiva de la organización del trabajo, como Arenas (1999) y Gálvez (2000b), o especificamente sobre una fábrica, como en el caso de Candela (1997) y Alonso (1998). 
que estudian, en alguna medida más presentes en los documentos de trabajo $^{34}$. Este hecho da idea de lo periféricas que resultan las cuestiones laborales dentro de la historiografía de la empresa en España. Igualmente, en los estudios de historia laboral, aun cuando se hace uso de archivos de empresa, se observa lo lejano que resulta todavía el análisis de la estructura y estrategias de las empresas o del tamaño y alcance de sus actividades para los investigadores que se dedican a estudiar el trabajo dentro de las empresas ${ }^{35}$. Sin embargo, los aspectos laborales no tienen por qué constituir un mundo aparte dentro del análisis empresarial. El que esto ocurra se explica en parte por la desconfianza mutua que ambos grupos de investigadores manifiestan, fenómeno que bebe directamente del antagonismo que ha llevado siempre a la tradición sindical y a la patronal a entender las relaciones entre empleadores y empleados sistemáticamente en términos de conflicto. De hecho, no debe extrañar que uno de los primeros estados de la cuestión sobre la historia empresarial que se llevaron a cabo dentro de la historiografía española, viera el tratamiento de los aspectos laborales dentro de la historia empresarial como «literatura de denuncia» ${ }^{36}$. Esto está en parte relacionado con la identificación automática entre trabajo y sindicatos en la historiografía, aunque, como ha demostrado Gálvez basándose en la crítica que el feminismo ha hecho del análisis marxista, es posible integrar el factor trabajo dentro de la historia empresarial sin centrarse en los sindicatos e incluso criticándolos en su papel de interlocutores exclusivos $^{37}$. Sin embargo, por regla general, se sigue identificando a los trabajadores con los sindicatos a la hora de tratar su papel en las empresas, incluso cuando se tienen en cuenta el tamaño y escala de las empresas,

34 Llordén (1994); Hernández Andreu y García Ruiz (1994); Núñez y Segreto (1994) y Comín y Martín Aceña (1996).

35 Los únicos artículos en las colecciones de historia empresarial que tratan los aspectos laborales son los de Lozano (1996) y Ocampo y Peribáñez (1994). Los Encuentros de Historia Económica de las Relaciones Laborales, celebrados en Sevilla en 1997 y 1999 , están llamados a convertirse en el mayor foro de debate sobre la organización del trabajo en las empresas, pero aún son pocas las comunicaciones que tratan los aspectos laborales dentro de las empresas más allá del estudio de los sindicatos. Sobre las discrepancias y confluencias de ambas disciplinas ver Martínez Ruiz (1998).

36 Coll y Tortella (1992). Esta «denuncia» fue criticada por Valdaliso (1993), principalmente porque en ella no se aportaban ejemplos de lo que los autores consideraban como literatura de denuncia. La respuesta de Coll (1993) a tal crítica fue la de argumentar que el revuelo causado con el artículo era ya bastante como para, además, citar los trabajos que ellos consideraban como literatura de denuncia.

37 Gálvez (2000b). 
su organización y estrategia ${ }^{38}$. Quizás sea ésa la causa por la que las cuestiones relacionadas con el asociacionismo patronal han encontrado mejor acogida dentro de la disciplina que las relacionadas con el asociacionismo obrero. Así, puesto que el asociacionismo patronal es en parte una respuesta al asociacionismo obrero y a la naturaleza de las relaciones Estado-empresa, estos trabajos arrojan información muy valiosa sobre los aspectos laborales en relación con la empresa ${ }^{39}$.

El segundo conjunto de causas está relacionado con el reparto de competencias en cuestiones laborales entre el Estado y las empresas a lo largo del siglo $\mathrm{xx}^{40}$. Se pretende demostrar que hasta recientemente, y particularmente en el caso español, las competencias estatales en materia laboral han sido muy fuertes. Esto puede explicar, por un lado, la falta de interés de los empresarios españoles a la hora de introducir métodos de organización del trabajo, además de los motivos ya expuestos sobre el miedo a la revuelta social o la falta de preparación de los gerentes para adaptar estos métodos, y, por otro, explicar por qué las cuestiones laborales en el seno de las empresas han sido tratadas en la mayoría de los casos por investigadores ajenos a la historia económica de la empresa. Este argumento sería consecuente con el hecho de que los modernistas han sabido integrar de forma más efectiva el trabajo dentro de los estudios empresariales, puesto que el factor trabajo era entonces más central al proceso productivo de lo que lo fue posteriormente, cuando las innovaciones tecnológicas y organizativas empezaron a basarse en la producción en masa con trabajadores no cualificados, aunque, como se ha apuntado, no siempre. La intromisión estatal, tal y como se ha expuesto en la primera parte, fue restringida hasta mediados del siglo XIX y no comenzó a ser realmente determinante hasta el período de entreguerras - principalmente en los regimenes fascistas - y, sobre todo, a partir de la posguerra y la difusión del

3k Zaldúa González (1998) realiza un interesante estudio sobre costes y relaciones laborales en La Papelera Española, S. A., uniendo las cuestiones laborales con la organización empresarial, las fluctuaciones en el mercado y el cambio tecnológico, pero aún entiende aquéllas de forma muy normativa.

39 Véase Cabrera y Del Rey (1996), o Linz y De Miguel (1966).

40 La competencia entre el Estado y las empresas en España ya fue señalada por Nadal (1975) con respecto a la financiación, ya que el Estado no vacilaba en competir con los empresarios del propio país, Nadal (1975), p. 28. Véase también el artículo de Gómez Mendoza y San Román (1997) sobre la competencia entre la empresa pública y privada. En este sentido, también es interesante la visión de Carreras, Tafuneil y Torres (1998) sobre el desarrollo inverso de la presencia de multinacionales en España y la empresa pública. Sobre el reparto, en este caso, de beneficios entre el Estado y las empresas, véase el caso del tabaco tratado por Gálvez (1999). 
Estado de bienestar. La duración del régimen de Franco y la gradual transición que posteriormente se vivió explican por qué en España la intromisión estatal en materia laboral ha sido mayor que en otros paises $o$, cuanto menos, distinta. De hecho, puede que haya sido la cuestión social y no la organización del trabajo en las empresas lo que haya importado en la España del siglo xx. No obstante, el hecho de que en la actualidad se esté viviendo otra revolución tecnológica basada en el capital humano serviría para explicar el creciente interés, tanto empresarial como académico, sobre el papel del trabajo dentro la empresa. La creciente importancia de la empresa privada y los mecanismos de mercado en las dos últimas décadas del siglo $\mathrm{xx}$ ha ido acompañada por el gradual abandono del Estado de actividades empresariales (desregulación, privatización y una gestión más eficiente, más privada de las empresas públicas). Igualmente, la importancia de la empresa en el mundo actual ha provocado un desplazamiento del centro de gravedad de los estudios laborales hacia la empresa, sus objetivos y capacidades, lo que ha propiciado que los historiadores de la empresa hayan comenzado a ofrecer su visión de la evolución de la organización del trabajo ${ }^{41}$. No obstante, el peso que ha tenido el intervencionismo estatal en la vida económica española a lo largo del siglo $\mathrm{xx}$, incluyendo la intromisión en la organización del trabajo en las empresas, hace del español un caso especialmente relevante. En este sentido, la historia económica de la empresa tiene mucho que aportar a los actuales debates sobre privatización y desregulación ${ }^{42}$.

El estudio del reparto de competencias entre el Estado y las empresas en materia laboral convierte al factor trabajo en un vehículo privilegiado de análisis de las relaciones Estado-empresa. Dentro de las cuestiones laborales, hay que considerar al Estado en un triple papel, el de empleador, el de regulador y el de intermediario. El peso de la empresa pública en la historia de España ha sido especialmente importante; por tanto, el estudio del Estado como empleador presenta muchas posibilidades para los historiadores económicos de la empresa en España, y permite conocer, a través del análisis histórico, la capacidad de adaptación de las empresas a su medio. Como empleadoras, las empresas públicas o concesionarias com-

${ }^{41}$ Fitzgerald (1999), p. 167. Además, muchos historiadores sociales se encuentran actualmente en el proceso de destacar al Estado como actor principal en lo que denominan Historia Social de la Política, Rivera (1998).

42 Sobre el auge y caída de la empresa pública, ver los artículos recopilados por Toninelli (2000). Kipping (2001), p. 3, argumenta que una de las tareas del historiador es la de demostrar el importante papel desempeñado por el Estado en el mundo empresarial, responsabilidad mayor de la que se le atribuye entre los economistas. 
parten una cierta responsabilidad social con el Estado, y, por tanto, están sujetas a un tipo de restricciones que las empresas privadas no sufren. De hecho, dentro de la teoría de la agencia, los problemas de agencia que se crean en estas empresas tienen la particularidad de que el principal representa los heterogéneos intereses de la ciudadanía. En este sentido, Gálvez y Comín argumentan, basándose en el ejemplo de la Compañía Arrendataria de Tabacos, que fue en la gestión laboral donde las empresas públicas o gestoras de algún bien público, como era el caso del monopolio de tabacos, vieron su libertad de gestión más constreñida, lo que les obligó, por estar sujetas además a un mayor escrutinio público, a ser más estratégicas en su gestión laboral a la hora, por ejemplo, de expulsar personal, con las consecuencias que ello conllevaba para la introducción de nueva tecnología y la modernización de la industria ${ }^{43}$. Destaca, pues, la poca atención que los aspectos relacionados con la organización del trabajo y los de acumulación de conocimientos y capacidades de los asalariados han despertado en los más importantes estudios sobre el INI, teniendo en cuenta la importancia que el régimen franquista concedía al pleno empleo y su relación con uno de los problemas estructurales más importantes de la economía española, el del paro ${ }^{44}$.

Pero la intervención del Estado en la organización de las empresas no sólo se da en las empresas públicas. Por ejemplo, otras grandes empresas como las de ferrocarriles están siendo ahora objeto de estudios que enfatizan la importancia de tratar los aspectos laborales, ya que durante mucho tiempo estas empresas sufrieron, a modo de regulación, una fuerte intervención estatal que enfatizaba sus obligaciones de servicio público e implicaba graves restricciones en la gestión laboral, llegándose en casos como el español a la militarización de los ferrocarriles ${ }^{45}$. Es interesante ver cómo una de las estrategias que este tipo de empresa desarrolló desde muy pronto fue la internalización de los mercados de trabajo que se desarrolló, en muchos casos, de la mano de las prácticas paternalistas que ya se han señalado como una constante en la organización del trabajo en las empresas

${ }^{43}$ Gálvez y Comín (2000)

${ }^{44}$ Los aspectos laborales no están presentes en los trabajos de Schwartz y González (1978), Martín Aceña y Comín (1991) y San Román (1999) sobre el INI. Algunos estudios particulares sobre algunas de las empresas del INI insisten en los aspectos laborales, como Martínez Ruiz (1999) sobre la Sociedad Anónima de las Construcciones Agricolas en Andalucía, y Bogaerts (2000) sobre el mundo social de ENSIDESA en Asturias.

Comín, Martín Aceña, Muñoz y Vidal (1998), vol. 1, pp. 195-201. Sobre la organización del trabajo en los ferrocarriles véase Channon (1998) y Ballesteros y Martínez Vara (1999). 
españolas. No obstante, la regulación en materia laboral, que afectó a la organización de la producción y el trabajo dentro de las empresas, se llevó a cabo como consecuencia del desarrollo de una legislación laboral que, como se ha visto anteriormente, se intensificó tras la Segunda Guerra Mundial, con la intención de garantizar la paz social y unas relaciones laborales estables, ayudando, sin duda, a garantizar la confianza de los inversores, sobre todo de los extranjeros. Este elemento debe tenerse en cuenta a la hora de explicar la interacción entre crecimiento económico e inversión directa extranjera en España. De hecho, la relación empresa-trabajo-Estado también es fundamental a la hora de tratar un campo que está siendo una de las áreas de mayor expansión de la historiografía empresarial, el del estudio de las multinacionales. Los costes laborales y/o la disponibilidad de mano de obra cualificada para determinadas industrias han sido algunos de los mayores condicionantes de localización de multinacionales en los países de acogida. En lo que se refiere a España, aún queda por estudiar la evolución de la presencia de empresas extranjeras con respecto a las distintas condiciones de trabajo, y el papel que desempeñaron, durante el régimen franquista, un régimen juridico represivo y un excesivo intervencionismo estatal, junto con la institución del sistema de sindicatos verticales, en las pautas de inversión extranjera ${ }^{46}$. Carreras, Tafunell y Torres han identificado la evolución de la presencia de multinacionales en España en proporción inversa al desarrollo de la empresa pública, pero aún queda por estudiar hasta qué punto las relaciones laborales fueron un imán de atracción o, en su defecto, una causa de abandono o desinterés como consecuencia de la continua intervención estatal ${ }^{47}$.

El tercer conjunto de funciones realizadas por el Estado, a la hora de explicar las diferencias en los sistemas de organización en las empresas, ha sido el de promotor o difusor de conocimiento. La difusión de los sistemas de organización del trabajo y de gestión, en general relacionados

${ }^{46}$ Sobre todo teniendo en cuenta que existen trabajos en la historiografía empresarial americana, como el de Greenwald (1998), que insisten en las ventajas para las empresas y el desarrollo económico del consenso y de los sistemas de sindicalismo vertical en determinadas industrias, claro que bajo un régimen político democrático.

${ }^{47}$ Carreras, Tafunell y Torres (1998). Aunque las razones de localización de la multinacional Río Tinto no están relacionadas con aspectos laborales, sino con la ubicación de los recursos naturales, como en toda inversión directa extranjera en el sector primario, los aspectos laborales constituyeron una fuente de intromisión del gobiemo franquista en la gestión de la compañía. Para Gómez Mendoza (1994), pp. 317-318, la reglamentación del trabajo afectó, por lo general, de forma indirecta a RTC, salvo en excepciones como la que supuso la congelación de plantillas de la minería. 
con la difusión del conocimiento y la tecnología, es uno de los temas que se consideran ahora esenciales dentro de la historia empresarial ${ }^{48}$. Con anterioridad, toda la literatura dedicada a ello ha estado dominada por la sociología - no la relacionada con la tecnología-. La difusión internacional de la tecnología debe ser complementada con el estudio de la difusión de las prácticas de organización de la gestión y organización del trabajo; especialmente es así si los sistemas tecnológicos se entienden como sistemas tecno-laborales, puesto que los modelos de organización están fuertemente ligados con la ideología, las instituciones y el tejido industrial y empresarial dominante en cada país o región. En este sentido, se podría concluir con la idea de que los empresarios españoles habrian sido influenciados por un excesivo dirigismo estatal que procuraba organizarles las cuestiones laborales. De hecho, hay que tener en cuenta que la adopción de los distintos modelos y paradigmas de organización empresarial no sigue necesariamente la credibilidad científica y no está solamente determinada por factores tecno-económicos, ya que las circunstancias institucionales, como la mentalidad y la formación, y las actividades de los grupos profesionales, el papel del Estado y las actitudes de los trabajadores y, sobre todo, su capacidad de adaptar las innovaciones, ya sean técnicas u organizativas, a las condiciones de partida, son conductivas.

\section{CONCLUSIONES}

La recuperación de la empresa como protagonista del crecimiento y del cambio económico llevada a cabo por la teoría económica y, especialmente, por corrientes como la economía evolutiva o la economía neo-institucional, ha concedido un protagonismo sin precedentes a la historia empresarial. Dentro de la historiografía española, Santiago López ha insistido en que la mejor aproximación posible a la historia empresarial es el eclecticismo en relación con el uso de diversas teorías y enfoques, pero este eclecticismo no sólo debe ser considerado en el uso de teorias, sino en el de temas y protagonistas de la historia empresarial ${ }^{49}$. En los años noventa, los aspectos laborales han sido reconocidos por la historiografía de la empresa como un agente olvidado dentro de la producción académica de esta disciplina. Este olvido ha sido, si cabe, más pronunciado en el

48 El conocimiento es el tema central del congreso en Oslo del 2001 de la European Business History Association.

49 López (1995). 
caso español. Esto se explica por el tardío desarrollo de la historia empresarial en España y por el reparto de competencias entre el Estado español y las empresas durante el siglo $\mathrm{xx}$, en este caso, sin duda favorable al primero. El intervencionismo estatal desembocó en un paternalismo en cascada Estado-empresa-trabajadores como sistema imperante de organización de la producción. No obstante, la falta de estudios y observaciones invita a concluir con mucha cautela sobre la evolución de las formas de organización predominantes en las distintas regiones y sectores económicos en España, tanto en el sector público como en el privado.

El objetivo de este artículo ha sido el de reflexionar sobre la necesidad de crear un marco analítico específico que comprenda el trabajo como un factor esencial dentro del conjunto de relaciones internas y externas que definen la competitividad de las empresas y las estrategias que éstas desarrollaron para adaptarse al medio. En este sentido es necesario «contar con los trabajadores» en la línea de las propuestas integradas en el artículo y que se recogen a continuación a modo de sumario y como agenda futura de investigación: Primero, los estudios de historia económica de la empresa deben integrar el factor trabajo más allá del análisis del conflicto con los sindicatos, y considerarlo como un elemento que puede incentivar, impedir, facilitar, retrasar, y, sobre todo, adaptar a las distintas condiciones de partida el cambio tecnológico. De esta forma, se pone el énfasis en los factores de oferta, aunque sin olvidar su necesaria interrelación con los de demanda. Segundo, frente al concepto de trabajo como fuerza comprada y aplicada empleado por los historiadores económicos, es necesario estudiar, además, la evolución de las formas de organización del trabajo, especialmente los momentos de cambio, y las diferencias que se dan entre regiones, países o sectores. Tercero, frente al énfasis en el capital físico a la hora de explicar las fuentes de competitividad de las empresas, hay que incidir en la importancia del capital humano, y no sólo o no sobre todo, de los indicadores más comunes de capital humano como son la alfabetización y la educación formal, sino de las cualificaciones adquiridas en los talleres, las cualificaciones organizativas y de investigación. Cuarto, estos análisis siempre tienen que hacerse teniendo en cuenta las condiciones de partida de cada región o país, y el marco institucional y cultural en el que las empresas operaron y que determinaron sus estrategias de adaptación al medio. De esa forma, además, se ahondará en el conocimiento de los distintos mercados de trabajo, ya que no existió un único mercado laboral. Quinto, sólo si el estudio de la organización del trabajo es integrado en las investigaciones que se realicen, se podrán constatar o refutar algunas de las 
ideas aquí apuntadas, y de esa forma, se podrán realizar generalizaciones que permitan introducir la organización del trabajo dentro de los modelos de cálculo de la productividad total de los factores. $Y$, por tanto, se podrá mejorar el conocimiento de las diferencias en la productividad. Sexto y último, el Estado debe ser considerado un actor fundamental a la hora de estudiar la evolución de la organización del trabajo dentro de las empresas en su triple papel de empleador, regulador e intermediario en la transmisión de cualificaciones y conocimientos. Además, de esta forma, la introducción del factor trabajo dentro de la historia económica de la empresa se convierte en un vehículo de análisis privilegiado para estudiar las relaciones Estado-empresas, sobre lo que se está debatiendo insistentemente en la sociedad actual. El hecho de que España haya pasado cuarenta años bajo una dictadura cuando otros países de su entorno estaban organizados bajo un sistema democrático y se consolidaba el modelo del Estado bienestar, hace de España un caso particularmente interesante.

\section{BIBLIOGRAFÍA}

Arenas, C.; Florencio, A., y Martinez, J. I. (eds.) (1998): Mercado de trabajo $y$ organización del trabajo en España (siglos XIX y XX), Sevilla, Universidad de Sevilla.

Aubanell, A. (1998): «Bienestar industrial en la empresa eléctrica madrileña en el primer tercio del siglo XX», en C. ARENAS et al. (eds.), Mercado de trabajo $y$ organización del trabajo en España (siglos XIX y XX), Sevilla, Universidad de Sevilla, pp. 141-160.

ARENAS, C. (1999): Empresa, mercados, mina y mineros. Río Tinto, 1873-1936, Huelva, Universidad de Huelva.

Alonso, L. (1998): As Tecedeiras do fume, Vigo, Edicions A Nosa Terra.

BABIANO, J. (1998): Paternalismo industrial y disciplina fabril en España (1938-1958), Madrid, CES.

Ballesteros, E., y MARTínez VARA, T. (1999): «El mosaico de las profesiones ferroviarias. El caso de la compañía de Madrid a Zaragoza y Alicante, 1910-1936», Segundo Encuentro de Historia de las Relaciones Laborales, Sevilla.

BECATINI, G. (ed.) (1987): Mercato e forze locali: Il distretto industriale, Bologna, Il Mulino.

BOGAERTS, J. (2000): El mundo social de ENSIDESA. Estado y patemalismo industrial (1950-1973), Avilés, Editorial Azucel.

Bowden, S., y Higgins, D. M. (1999): «Productivity on the Cheap? The "More Looms" Experiment and the Lancashire Weaving Industry during the Inter-War Years», Business History, 41, 3, pp. 21-41.

BROADBERRY, S. N. (1997): The Productivity Race. British Manufacturing in International Perspective, 1850-1990, New York, Cambridge University Press. 
BRUTON, K. (1994): The Business Culture in Spain, Oxford, Butterworth Heinemann.

BuesA, M., y Molero, J. (1982): «Cambio tecnológico y proceso de trabajo: una aproximación al papel del Estado en la introducción de los métodos de la organización científica del trabajo en la economía española durante los años cincuenta», Revista de Trabajo, vol. 2, pp. 249-268.

CABrera, M., y DEL REY, F. (1996): «Los intereses económicos organizados en España. Un siglo en la historia del asociacionismo empresarial», en F. Comín y P. MaRtín ACEÑa (eds.), La empresa en la bistoria de España, Madrid, Civitas, pp. 442-456.

CANDELA, P. (1997): Trabajo y vida en la experiencia laboral de las cigarreras madrileñas, 1888-1927, Madrid, Tecnos.

Carreras, A., y TAfunell, X. (1997): «Spain: Big Manufacturing Firms between the State and the Market, 1917-1990», en A. Chandier, F. Amatori y T. Hixt. No (eds.), Big Business and the Wealth of Nations, Cambridge, Cambridge University Press, pp. 277-304.

Carreras, A.; Tafunell, X., y Torres, E. (1998): «Against Integration. The Rise and Decline of Spanish State-owned Firms and the Decline and Rise of Multinationals, 1939-1990», en U. Olsson (ed.), Business and European Integration since 1800. Regional, National and International Perspectives, Göteborg, Meddelanden Fran Ekonomisk-Historiska Institutionen, pp. 31-48.

CASTILlo, J. J. (1996): «¿Ha habido en España organizadores de la producción? Entre dos congresos de ingeniería, 1919-1950», en S. CASTILlo (coord.), El trabajo a través de la Historia, Madrid, Asociación de Historia Social, pp. 233-264.

Coll, S. (1993): «El profesor Valdaliso, a vueltas con la historia de la empresa», Revista de Historia Económica, 11, 3, pp. 627-630.

Coll, S., y TORTElla, G. (1992): «Reflexiones sobre la historia empresarial: estado de la cuestión en España», Información Comercial Española, 708-709, pp. 13-24.

COMín, F., y Martin ACEÑA, P. (eds.) (1996): La empresa en la bistoria de España, Madrid, Civitas,

COMIN, F., y MARTín ACEÑ, P. (1999): Tabacalera y el estanco del tabaco en España, 1636-1998, Madrid, Fundación Tabacalera.

Comín, F.; Martín-Aceña, P.; Muñoz, M., y Vidal, J. (1998): 150 años de bistoria de los ferrocarriles españoles, Madrid, Anaya-Fundación de los Ferrocarriles Españoles.

Chandler, A.; Amator, F., y Hixino, T. (eds.) (1997): Big Business and the Wealth of Nations, Cambridge, Cambridge University Press.

Channon, G. (1998): «Railway Labor Management Policies in Britain and United States between 1890 and 1920m, en J. Amstrong, C. Bouneau y J. Vidal (eds.), Railway Management and its Organisational Structure: its Impact on and Diffusion into the General Economy, Madrid, Fundación Fomento de la Historia Económica, pp. 91-102.

De Miguel, A., y Linz, J. (1967): «Los problemas de la retribución y del rendimiento vistos por los empresarios españoles», Revista del Trabajo, marzo, pp. 35-140. 
FITZGERALD, R. (1999): «Employment Relations and Industrial Welfare in Britain: Business Ethics versus Labor Markets», Business and Economic History, 28, 2, pp. 167-179.

FitzGerald, R., y Rowley, C. (eds.) (1997): Human Resources and the Firm in International Perspective, Cheltenham, Edward Elgar.

GALVEZ, L. (1999): «Companies, Governments, Consumers and Tobacco Addiction: the Spanish case 1880s-1930s», Business and Economic History, vol. 42, pp. $87-128$.

- (2000a): «Género y cambio tecnológico. Rentabilidad económica y política del proceso de industrialización del monopolio de tabacos en España (1887-1945)», Revista de Historia Económica, 18, 1, pp. 11-45.

- (2000b): La Compañia Arrendataria de Tabacos 1887-1945: Cambio Tecnológico y Empleo Femenino, Madrid, LID.

GálvEZ, L., y COMín, F. (2000): «Business and Government: Labour Management in the Spanish Tobacco Monopoly (1887-1935)», en A. M. KuiJLAars et al. (eds.), Business and Society, Rotterdam, CBG, pp. 159-170.

GIL, J. (1967): «Los Estados Unidos en el movimiento de la productividad», Información Comercial Española, sept., pp. 145-9.

Gómez Mendoza, A. (1994): El Gibraltar Económico. Franco y Río Tinto, 1936-1954, Madrid, Civitas.

Gómez Mendoza, A., y SAN Román, E. (1997): «Competition between Private and Public Enterprise in Spain, 1939-1959: An Alternative View», Business and Economic History, 26, 2, pp. 696-708.

Guillén, M. (1994): Models of Management. Work Autbority, and Organization in a Comparative Perspective, Chicago, The University of Chicago Press.

GreEnwaLd, R. A. (1998): «More than a Strike: Ethnicity, Labor Relations, and the Origins of the Protocol of Peace in the New York Ladies' Garment Industry», Business and Economic History, 27, 2, pp. 318-329.

Hernandez Andreu, J., y Garcia Ruiz, J. L. (eds.) (1994): Lecturas de bistoria empresarial, Madrid, Civitas.

Houpr, S., y Ortaz-VIllajos, J. M. (dir.) (1998): Astilleros Españoles, 1872-1998. La construcción naval en España, Madrid, LID.

JONES, G. G. (1997): «Great Britain: Big Business, Management and Competitiveness in xxth Century Britain», en A. Chandler, F. Amatori y T. Hikino (eds.), Big Business and the Wealth of Nations, Cambridge, Cambridge University Press, pp. 102-138.

KIPPING, M. (2001): «Development and Dynamics of Government-Business Relations», en F. Amatori y G. G. Jones (eds.), Business History Around the World at the Turn of the Century, New York, Cambridge University Press.

LAZONICK, W. (1990): Competitive Advantage on the Shop Floor, Cambrigde, Mass., Harvard University Press.

Lipartito, K. (1994): «When Women Were Switches: Technology, Work and Gender in the Telephone Industry, 1890-1920», American Historical Review, 99, 4, pp. 1075-1111.

Linz, J., y DE Miguel, A. (1966): Los empresarios ante el poder público, Madrid, Centro de Estudios Constitucionales. 
LLORDÉN, M. (comp.) (1994): De empresas y empresarios en la España contemporánea, Universidad de Oviedo, Servicio de Publicaciones.

LOPEZ, S. (1995): «La Historia Económica de la Empresa», en IV Encuentro de Didáctica de la Historia Económica, Departamento de Economía, Universidad Pública de Navarra, pp. 45-69.

López S., y VALDAliso, J. M. (eds.) (1997): ¿Que inventen ellos? Tecnología, empresa y cambio económico en la España contemporánea, Madrid, Alianza.

LozANO, A. (1996): «De empresa pública a empresa privada: la gestión de los arsenales del Estado», en F. Comín, y P. MARTín ACEÑa (eds.), La empresa en la bistoria de España, Madrid, Civitas, pp. 369-382.

- (1997): «Estado, importación de tecnología y nacionalización de la construcción naval militar española: la SECN, 1909-1935», en S. LóPEZ y J. M. VALDALISO (eds.), ¿Que inventen ellos? Tecnología, empresa y cambio económico en la España contemporánea, Madrid, Alianza, pp. 281-303.

Malo OCAÑA, M. A. (1998): «Una aproximación al coste del despido individual en la Dictadura y en la Democracia», en C. Arenas et al. (eds.), Mercado de Trabajo y Organización del Trabajo en España (siglos XIX y XX), Sevilla, Universidad de Sevilla, pp. 89-100.

Martín ACEña, P., y Comín, F. (1991): INI. 50 Años de Industrialización en España, Madrid, Espasa-Calpe.

MarTínez Ruiz, J. I. (1998): «La historia económica de las relaciones laborales, nueva especialización disciplinar», en C. Arenas et al. (eds.), Mercado de Trabajo y Organización del Trabajo en España (siglos XIX y XX), Sevilla, Universidad de Sevilla, pp. 23-40.

- (1999): «Privatización de empresas públicas y desindustrialización en Andalucía: la Sociedad Anónima de Construcciones Agrícolas, 1964-1972», en A. PAREJo y A. SÁnchez (eds.), Economía andaluza e Historia Industrial. Estudios en bomenaje a Jordi Nadal, Motril, Asukaría Mediterránea, pp. 405-414.

MOKYR, J. (1990): The Lever of Riches. Technological Creativity and Economic Progress, Oxford, Oxford University Press.

MuNOZ ABELEDO, L. (1999): «Las relaciones laborales y la estructura del mercado de trabajo en la industria de conservas de pescado durante el franquismo». Segundo Encuentro de Historia de las Relaciones Laborales, Sevilla.

NADAL, J. (1975): El fracaso de la revolución industrial en España (1814-1913), Barcelona, Ariel.

NúNEZ, C. E. (1992): La fuente de la riqueza. Educación y desarrollo económico en la España contemporánea, Madrid, Alianza.

NúNEZ, G., y SEGRETTO, L. (eds.) (1994): Introducción a la bistoria de la empresa en España, Madrid, Abacus.

OCAMPO J., y PERIBÁÑEZ, D. (1994): «Empresas y trabajadores en la industrialización asturiana del siglo XIX», en M. LLORDÉN (comp.), De empresas y empresarios en la España contemporánea, Universidad de Oviedo, Servicio de Publicaciones, pp. 101-117.

RIERA, S. (1993): «Industrialization and Technical Education in Spain, 1850-1914», en R. Fox y A. GUAGNINI (eds.), Education, Technology and Industrial Performance in Europe, 1850-1939, Cambridge, Cambridge University Press, pp. 141-170. 
Rivera, A. (1998): «Orden social, reforma social, estado social», en S. CASTTLLO y J. M. OrTtz (coords.), Estado, protesta y movimientos sociales, Bilbao, Asociación de Historia Social, pp. 3-18.

Rosés, J. (1997): «La integración vertical en la industria algodonera catalana», en S. López y J. M. VALDALISO (eds.), ¿Que inventen ellos? Tecnología, empresa y cambio económico en la España contemporánea, Madrid, Alianza, pp. 249-80.

SAN Román, E. (1999): Ejército e Industria. El nacimiento del INI, Barcelona, Crítica. SCranton, P. (1991): «Diversity in Diversity», Business History Review, 57, pp. $27-90$.

Scranton, P., y Horowtrz, R. (1997): «The Future of Business History: an Introduction», Business and Economic History, 26, 1, pp. 1-4.

SChWARTZ, P., y GonZÁlez, M. J. (1978): Una bistoria del Instituto Nacional de Industria, Madrid, Tecnos.

SEGReTtO, L. (1994): «De la historia económica a la historia de la empresa: la marcha del debate en España», en G. NúNEZ y L. SEGRETTO (eds.), Introducción a la bistoria de la empresa en España, Madrid, Abacus, pp. 17-37.

Tiratsoo, N., y Tomlinson, J. (1994): «Restrictive Practices on the Shop Floor in Britain, 1945-60: Myth and Reality», Business History, 36, 2, pp. 65-82.

Tolliday S., y ZeItuIn, J. (1991): The Power to Manage? Employers and Industrial Relations in Comparative-Historical Perspective, London, Routlege.

Toninelil, P. A. (ed.) (2000): The Rise and Fall of State-Owned Enterprise in the Western World, New York, Cambridge University Press.

Torras, J. (1993): «Gremio, familia y cambio tecnológico. Pelaires y tejedores en Igualada, 1695-1765", Revista de Historia Industrial, 2, pp. 11-30.

VALDALISO, J. M. (1993): «Algunas reflexiones acerca de la Historia empresarial y su desarrollo en España», Revista de Historia Económica, 11, 2, pp. 417-433.

VAlDALiso, J. M. (1997) [1999]: «La historia empresarial en España. Origenes, desarrollo y controversias respecto a una nueva disciplina», America Latina en la Historia Económica. Boletín de Fuentes, 7, pp. 103-132.

VAlDaliso, J. M., y López, S. (2000): Historia económica de la empresa, Barcelona, Crítica.

Zaldúa GONZÁleZ, I. (1998): «Costes y relaciones laborales en La Papelera espanola, S. A., 1902-1959», en C. ArEnAS et al. (eds.), Mercado de trabajo y organización del trabajo en España (siglos XIX y XX), Sevilla, Universidad de Sevilla, pp. 123-138. 